\title{
Biometrics in Practice: What Does HCI Have to Say?
}

\author{
Lynne Coventry ${ }^{1}$, Graham I. Johnson ${ }^{2}$, Tom McEwan ${ }^{3}$, and Chris Riley ${ }^{2}$ \\ ${ }^{1}$ Johnston and Johnston Ltd. \\ ${ }^{2}$ NCR Labs, Dundee \\ christopher.riley@ncr.com \\ ${ }^{3}$ Edinburgh Napier University
}

\begin{abstract}
This panel discusses biometric technologies from different perspectives in $\mathrm{HCI}$ in order to arrive at a coherent response that the community can give in this area. The challenges of doing this will also be of interest in terms of HCI's general influence on topical issues.
\end{abstract}

Keywords: Biometrics; public influence of HCI.

\section{Introduction}

Biometrics pose a challenge to $\mathrm{HCI}$, in that they expose many of the contradictions inherent in defining our discipline. For at least ten years our community has debated a range of issues - from the minutiae of the capture technology itself to the wider scale of socio-technical analysis - yet the HCI community finds it difficult to communicate best practice to the outside world. In part this is because of the different lenses through which a topical and provocative subject can be viewed - cultural context of use, accuracy of authentication, interaction design, user habituation, humancenteredness $\mathrm{v}$ mandatory use. This panel debates what the HCI community can offer to policy makers, manufacturers and deplorers of biometric systems and in so-doing sheds light on how the HCI community can be more influential in topical debates. The panellists represent a range of stakeholders: Lynne Coventry has many years experience evaluating biometrics and usability, Karen Renaud has experience working with numerous different authentication systems. Tom McEwan researches human-centred innovation and seeks to help the UK's HCI community give a coherent response to topical issues such as biometrics and Chris Riley is well placed to represent the views of systems integrators and deplorers of biometrics. Finally, Linda Sorensen can give a perspective from NPL, which has advised on biometrics for many years. Each panellist will speak on their recent practical experiences with biometrics, contrast this with HCI theory and address the following questions:

- What are the characteristics of an acceptable biometric authentication system?

- Taking biometrics as an example, has HCI anything to offer beyond the traditional domains of systems and interface design?

- Are there areas that the HCI community regard as outside the field, e.g. the creation of information media designed to make biometrics acceptable? 
- What ethical contradictions are there in using human-centred methodologies to design a mandatory biometric system?

Biometric authentication is the process of identifying an individual through measurable characteristics of their behaviour, anatomy or physiology and is becoming increasingly pervasive in modern society. However, biometrics are a contentious form of personal identification, described as an ethically challenging technology which undermines personal privacy [1]. When studied, people voice concern about the technology or the way the way it will be used $[2,3]$, and with some justification. The use of biometrics automates the identification process, removing control from users, eliminating interpersonal trust. The characteristics used during biometric authentication, such as fingerprints or iris patterns, cannot simply be re-issued if they become compromised.

The HCI community has increasingly turned its attention to the usability and acceptability of biometrics and security systems, for example in the design of the biometric user interface [4], the overall biometric system [5] and the context within which biometrics are used [6]. A recent workshop on the usability of biometric systems [7] is further evidence of an increased focus on biometrics from the HCI community. Given the prevalence and impact of public data security breaches, making biometrics acceptable would seem to be a hard sell. Should the HCI community help with this sell? Can human-centred methods help design with mandatory biometric systems that appear to have no direct benefit for those required to use them (for example the US-VISIT programme and the proposed UK Identity Card scheme). Is user is only really at the centre of the design process, when speed and accuracy of the system are primary design goals?

\section{References}

1. Alterman, A.: “A piece of yourself": Ethical issues in biometric identification. Ethics and Information Technology 5, 139-150 (2003)

2. BioSec Consortium.: Report on results of first phase usability testing and guidelines for developers (2004)

3. Riley, C., Buckner, K., Johnson, G.I., Benyon, D.: Culture \& Biometrics: Regional differences in the perception of biometric authentication technologies. Journal of AI \& Society (2009)

4. Theofanos, M., Michaels, R., Scholtz, J., Morse, E., May, P.: Does Habituation Affect Fingerprint Quality? Paper presented at the 24th SIGCHI Conference of Human Factors in Computing Systems, Canada (2006)

5. Briggs, P., Oliver, P.: Biometric daemons: authentication via electronic pets. Paper presented at the 26th SIGCHI Conference on Human Factors in Computing Systems, Florence, Italy (2008)

6. Heckle, R., Patrick, A., Ozok, A.: Perception and Acceptance of Fingerprint Biometric Technology. Paper presented at the Symposium on Usable Privacy and Security (SOUPS), Pittsburgh (2007)

7. Herman, M.: Objectives and Outcomes. In: The NIST International Workshop on Usability and Biometrics, Washington, June 23-24 (2008) 